
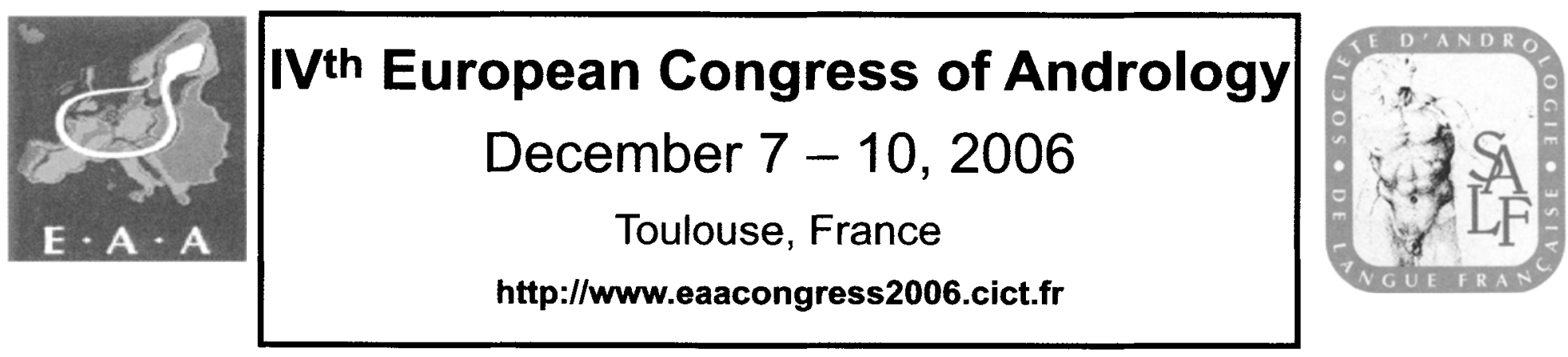

In recent decades, Andrology has been able to develop a remarkable and holistic approach to various aspects of male reproductive health.

Infertility, contraception, sexuality, embryonic stem cells, testis and prostate cancer, premature ejaculation and erectile dysfunction are all fields where substantial fundamental and clinical progress have been made.

The next major event in andrology will be

\title{
the IVth European Congress of Andrology
}

to be held from December 7-10, 2006, in Toulouse

\section{Toulouse}

a delightful southern European city, with a unique blend of tradition (rugby, cassoulet and foie gras) and modernity (Airbus) with good access from all corners of Europe

\section{Submission of abstracts}

\section{Scientific programme (see behind)}

The deadline with obligatory registration, is July 14, 2006

Up to 36 abstracts will be selected for an oral presentation in plenary sessions

Registration will be possible through the congress website.

Fees EAA and SALF members: 350 euros, Non-members: 400 euros, Students: 200 euros

Accommodation: a list of typical hotels located in the heart of the city and near the congress site, and offering special rates to participants will be provided with on-line registration. 
'Basic Science and Clinical Practice Interphase in Andrology'

IVth workshop on Epididymis in Chatel-Guyon (Puy de Dome ; France); December 4 to $6^{\text {th }}$; see http://www.agence-mo.com/epididymis 4

Session SALF (The French Society of Andrology) (December, the 7 th; 14-18h) 14h - 16h30 SYNDROME doulouREUX PELVIEN CHEZ L'HOMME ; modérateurs: D Delavierre \& O Rampin 14h - 14h20 Dr Jean Jacques Labat: Physiopathologie du syndrome douloureux pelvien chronique 14h20 - 14h40 Dr Christian Baude : Thérapeutique du syndrome douloureux pelvien chronique 14h40 - 15h00 Dr Pierre Bondil : Dyspareunie chez l'homme 15h - 15h30 Discussion générale sur le syndrome douloureux pelvien chronique chez l'homme

\section{IVth European Congress of Andrology (December 7-10th) - PLENARY SESSIONS}

E. Nieschlag, Germany (Opening Lecture): History of the testis and androgen treatment (Chair: F. Wu)

J. Trasler, Canada: Reproductive epigenetics and transgenerational toxicity (Chair: A. Grootegoed)

M. Maggi, Italy: Pathophysiology of erectile dysfunction (Chair: A. Lenzi)

P. Sassone-Corsi, France: Gene regulation in spermatogenesis and spermiogenesis insights and implications for human infertility (Chair: E. Baldi)

M. Stefannini, Italy: Gametogenic stem cells. (Chair: G. Verhoeven)

A. Sutcliffe, UK: Health of children conceived through intracytoplasmic sperm injection, in vitro fertilization, and natural conception (Chair: B. Zorn)

\section{Symposia}

\section{Prostate (Chair: F. Hamdy/W. Weidner)}

F. Hamdy, U.K: Early diagnosis and management of prostate cancer

M. Wolf, Finland: Microarray-based molecular profiling of hormone-refractory prostate cancer

A. Poletti, Italy: Role of oestrogen receptor ? in the pathogenesis and progression of prostate cancer

J. Fitzpatrick, Eire: Benign prostatic hyperplasia - current management options and future prospects

Testis development, cryptorchidism, and cancer (Chair: $P$. Thonneau/N. Jorgensen)

E. Rajpert-De Meyts, Denmark: Germ cell cancer - clinical applications of gene expression profiles

M. Benahmed, France: Fetal androgen disruption and chronic adult germ cell apoptosis -

a model for the testicular dysgenesis syndrome

E. Huyghe, France: Management of testicular cancer with focus on fertility after treatments

N. Agoulnik, USA: Cryptorchidism - relaxins and other factors

Spermatogenesis and spermiogenesis (Chair: D. Royere/R. Mieusset)

S. Rousseaux, France: The role of chromatin in the establishment of paternal epigenetic information during spermatogenesis

M. Mark, France: Retinoic acid metabolism and signaling in the adult and developing testis

M. Bergmann, Germany: Connexins - relevance to rodent and human spermatogenesis?

M. Primig, Switzerland: Databases and data mining in studies on spermatogenesis

Spermatozoa maturation and function (Chair: T. Cooper/M Bailly)

R. Sullivan, Canada: Human epididymal proteins - regulation and interactions with spermatozoa

J-L. Dacheux, France: Human epididymal proteins - secretion, absorption, and luminal fluid composition

R. Jones, UK: Sperm lipid dynamics - maturation and function

S. Publicover, UK: Sperm calcium channel signalling - nature and function

Premature ejaculation (Chair: E. Jannini/G. Forti)

M. Waldinger, Netherlands: Definition and epidemiology of premature ejaculation

A. Lenzi, Italy: Pathogenesis and diagnosis of premature ejaculation

F. Giuliano, France: Management of premature ejaculation

Genital tract infections (Chair: B. Zorn/L. Bujan)

N. Dejucq-Rainsford, France: Antiviral defences of the testis

C. Pasquier, France: Virus and semen

C. Gillling-Smith, UK: Assisted reproductive technology in couples with HIV-infected male partners

W. Eggert-Kruse, Germany: Bacterial male genital tract infection and sperm quality 


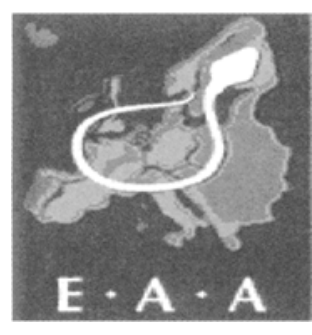

\section{4ème Congrès Européen d'Andrologie 23ème Congrès de la SALF 7 - 10 Décembre2006 Toulouse, France http://www.eaacongress2006.cict.fr}

\section{Bienvenue à Toulouse au IV Congrès Européen d'andrologie}

Au cours des dernières décennies, l'Andrologie a su développer une approche remarquable et holistique en reproduction humaine : infécondité et assistance médicale à la procréation, spermiologie et autoconservation de sperme, cancer du testicule et de la prostate, sexualité et qualité de vie de l'homme âgé, autant de champs où l'Andrologie a su allier recherche fondamentale et approche clinique.

Cette année, l'événement andrologique sera le IV Congrès de l'Académie Européenne d'Andrologie, qui se tiendra à Toulouse, du 7 au 10 décembre 2006, précédé, le jeudi 7 décembre de 14 à $17 \mathrm{~h}$ d'une session spécifique de la Société d'Andrologie de Langue Française (SALF). Par ailleurs et de manière satellite à ce congrès européen d'andrologie (inscription groupée), se tiendra à Chatel-Guyon (Puy de Dome), du 4 au 7 décembre, la 4eme réunion Internationale sur l'Epididyme, sous l'égide de I'ISAVEAADGA/BAS et de la SALF (voir http://www.agence-mo.com/epididymis4)

Aux aspects scientifiques, les organisateurs toulousains ont aussi associé tradition et qualité de vie du sud-ouest, match de rugby; visite d'Airbus; dégustation et achat de foie gras, cassoulet, Madiran et Tariquet.

\section{Un programme scientifique à l'interface entre science fondamentale et recherche clinique en andrologie}

Le Comité scientifique international et le Comité local d'organisation ont élaboré un programme scientifique (cf. verso) particulièrement attractif avec des communications sur des aspects cliniques et sur des recherches plus fondamentales. Ce congrès associera six séances plénières et six séances thématiques, suivies par la présentation, en plénière, des meilleurs travaux originaux sélectionnés parmi les résumés.

Par ailleurs, la session spécifique (en français) de la SALF, du jeudi 7 décembre de 14 à 17h., sera consacrée au « SYNDROME DOULOUREUX PELVIEN CHRONIQUE CHEZ L'HOMME ».

\section{Lieu du Congrès}

Ce congrès se tiendra à l' Hôtel Dieu-Saint Jacques, salle Gaspard-de-Maniban, 2 rue Viguerie, à Toulouse.

\section{Soumission des résumés}

Les 30 meilleurs résumés présenteront leurs travaux en séance plénière. Les autres résumés sélectionnés seront présentés lors de deux séances de poster, avec plusieurs prix des meilleurs posters.

Les résumés doivent impérativement être soumis le 14 juillet 2006 au plus tard, en respectant les indications présentées sur le site http://www.eaacongress2006.cict.fr, rubrique " soumission des résumés ".

\section{Inscription}

L'inscription se fait directement sur le site Web par carte de crédit, via le site sécurisé Paypal (ou par transfert bancaire). Voir le site Web, rublique «inscription». Le montant de l'inscription, avant le 15 juillet est de 350 Euro pour les membres EAA ou SALF, 400 Euro pour les non-membres, et 200 Euro pour les étudiants.

\section{Hébergement}

Une liste d'hôtels situés en centre ville, près du lieu du congrès et proposant des prix abordables a été sélectionnée. Pour réserver, consulter le site Web, rubrique « hébergement ".

\section{Visites touristiques}

En partenariat avec le Comité de Tourisme de Haute Garonne, des visites touristiques (matchs de rugby, marché du foie gras, visite d'Airbus) sont proposés (cf. site Web, rubrique " visite touristique "). 


\section{Programme Scientifique}

Session SALF (jeudi 7 décembre)

14h - 16h30 SYNDROME DOULOUREUX PELVIEN CHRONIQUE CHEZ L'HOMME ; modérateurs:

D. Delavierre \& O. Rampin

14h - 14h20 Dr Jean Jacques Labat: Physiopathologie du syndrome douloureux pelvien chronique 14h20 - 14h40 Dr Christian Baude : Prise en charge thérapeutique du syndrome douloureux pelvien chronique

14h40 - 15h Dr Pierre Bondil : Dyspareunie chez l'homme

15h - 15h30 Discussion générale sur le syndrome douloureux pelvien chronique chez l'homme 15h30 -16h30 Présentation des bourses et prix M2 et DESS de la SALF

\section{Congrès Européen d'andrologie (7 - 10 décembre) SÉANCES PLÉNIĖRES}

E. Nieschlag, Germany (Opening Lecture): History of the testis and androgen treatment (Chair: F. Wu)

J. Trasler, Canada: Reproductive epigenetics and transgenerational toxicity (Chair: A. Grootegoed)

M. Maggi, Italy: Pathophysiology of erectile dysfunction (Chair: A. Lenzi)

P. Sassone-Corsi, France: Gene regulation in spermatogenesis and spermiogenesis insights and implications for human infertility (Chair: E. Baldi)

M. Stefannini, Italy: Gametogenic stem cells. (Chair: G. Verhoeven)

A. Sutcliffe, UK: Health of children conceived through intracytoplasmic sperm injection, in vitro fertilization, and natural conception (Chair: B. Zorn)

6 Symposia (23 orateurs invités)

Prostate (Chair: F. Hamdy/W. Weidner)

F. Hamdy (U.K.): Early diagnosis and management of prostate cancer

M. Wolf (Finland): Microarray-based molecular profiling of hormone-refractory prostate cancer

A. Poletti (Italy): Role of oestrogen receptor? in the pathogenesis and progression of prostate cancer

J. Fitzpatrick (Eire): Benign prostatic hyperplasia - current management options and future prospects

Testis development, cryptorchidism, and cancer (Chair: P. Thonneau/N. Jorgensen)

E. Rajpert-De Meyts, Denmark: Germ cell cancer - clinical applications of gene expression profiles

M. Benahmed, France: Fetal androgen disruption and chronic adult germ cell apoptosis - a model for the testicular dysgenesis syndrome

E. Huyghe, France: Management of testicular cancer with focus on fertility after treatments

N. Agoulnik (USA): Cryptorchidism - relaxins and other factors

Spermatogenesis and spermiogenesis (Chair: D. Royere/R. Mieusset)

S. Rousseaux, France: The role of chromatin in the establishment of paternal epigenetic information during spermatogenesis

M. Mark, France: Retinoic acid metabolism and signaling in the adult and developing testis

M. Bergmann, Germany: Connexins - relevance to rodent and human spermatogenesis?

M. Primig, Switzerland: Databases and data mining in studies on spermatogenesis

Spermatozoa maturation and function (Chair: T. Cooper/M Bailly).

R. Sullivan, Canada: Human epididymal proteins - regulation and interactions with spermatozoa

J-L. Dacheux, France: Human epididymal proteins - secretion, absorption, and luminal fluid composition

R. Jones, UK: Sperm lipid dynamics - maturation and function

S. Publicover, UK: Sperm calcium channel signalling - nature and function

Premature ejaculation (Chair: E. Jannini/G. Forti)

M. Waldinger, Netherlands: Definition and epidemiology of premature ejaculation

A. Lenzi, Italy: Pathogenesis and diagnosis of premature ejaculation

F. Giuliano, France: Management of premature ejaculation

Genital tract infections (Chair: B. Zorn/L. Bujan)

N. Dejucq-Rainsford, France: Antiviral defences of the testis

C. Pasquier, France: Virus and semen

C. Gillling-Smith, UK: Assisted reproductive technology in couples with HIV-infected male partners

W. Eggert-Kruse, Germany: Bacterial male genital tract infection and sperm quality 